\title{
Impact of shot-peening on a single crystal nickel-based superalloy
}

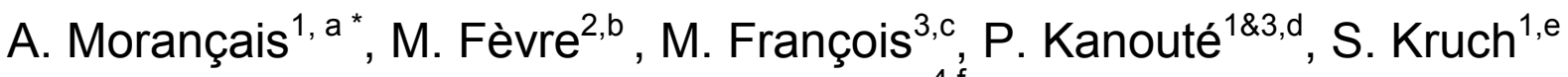 \\ and $\mathrm{A}$. Longuet ${ }^{4, f}$ \\ IONERA - The French Aerospace Lab, F-92322 Châtillon, France \\ 2LEM UMR 104 CNRS-ONERA, F-92322 Châtillon, France \\ ${ }^{3}$ ICD-LASMIS, UMR CNRS 6279 Université de Technologie de Troyes, 12 Rue Marie Curie, CS \\ 42060, 10004 Troyes, France \\ ${ }^{4}$ SAFRAN Snecma Villaroche, Rond-point René Ravaud, 77550 Réau, France \\ a*amelie.morancais@onera.fr, ${ }^{\mathrm{b}}$ mathieu.fevre@onera.fr, ${ }^{\mathrm{c}}$ manuel.francois@utt.fr, \\ dpascale.kanoute@onera.fr, ${ }^{\mathrm{e}}$ serge.kruch@onera.fr, ${ }^{\mathrm{f}}$ arnaud.longuet@snecma.fr
}

Keywords: Shot-peening, single crystal, residual stresses, X-ray diffraction.

\begin{abstract}
Shot-peening is used to improve the lifetime of mechanical components through the introduction of compressive residual stresses (RS) in a surface layer. In this study, we investigate the impact of such a pre-stressing treatment on a single crystal nickel-based superalloy for high pressure turbine blades of engine aircrafts. In addition to conventional metallographic tools used to characterize the alloy microstructure and the zone affected by shot-peening, X-ray measurements have been performed in order to determine residual stress depth profiles.
\end{abstract}

\section{Introduction}

High pressure turbine blades of aircraft engines are made of single crystal nickel-based superalloys. The blade is supported by a base which includes a platform, a fir-tree root which is put into a machined complementary shape in the periphery of the disk, and finally a stilt connecting the root and the platform. During the engine service, the fatigue of the root is mainly due to cyclic mechanical loading, temperature and vibrations. In order to improve the fatigue resistance, the root is shot-peened.

Many studies have already been carried out on polycrystalline superalloys for turbine disks in order to characterize residual stress (RS) levels introduced by shot-peening, their evolution with temperature and cyclic loading conditions and their influences on the disk life time [1]. Stress levels are usually determined with diffraction techniques (X-ray or neutrons) using the $\sin ^{2} \psi$ method. However, only few studies are devoted to shot-peened single crystal superalloys [2].

This present study is a part of a larger project on the influence of residual stresses introduced by shot-peening on the lifetime of the AM1 superalloy. The goal is to determine the stress profile in the depth of fatigue samples preliminary shot-peened. The residual stress depth profile can be introduced as an input parameter in calculations to estimate the life time of AM1 components exposed to complex thermo-mechanical loading. This paper focuses on the determination of RS depth profiles and the results obtained using X-ray diffraction measurements on flat samples are discussed.

The paper is organized as follows. In section 1, the investigated samples are described in terms of heat and mechanical treatments, crystallographic orientations, microstructures and phases. Section 2 presents the microhardness and EBSD characterizations and section 3, the residual stress determination using one of the methods initially developed by Ortner [3] and reformulated exclusively in the reciprocal lattice by François [4]. 


\section{Samples description}

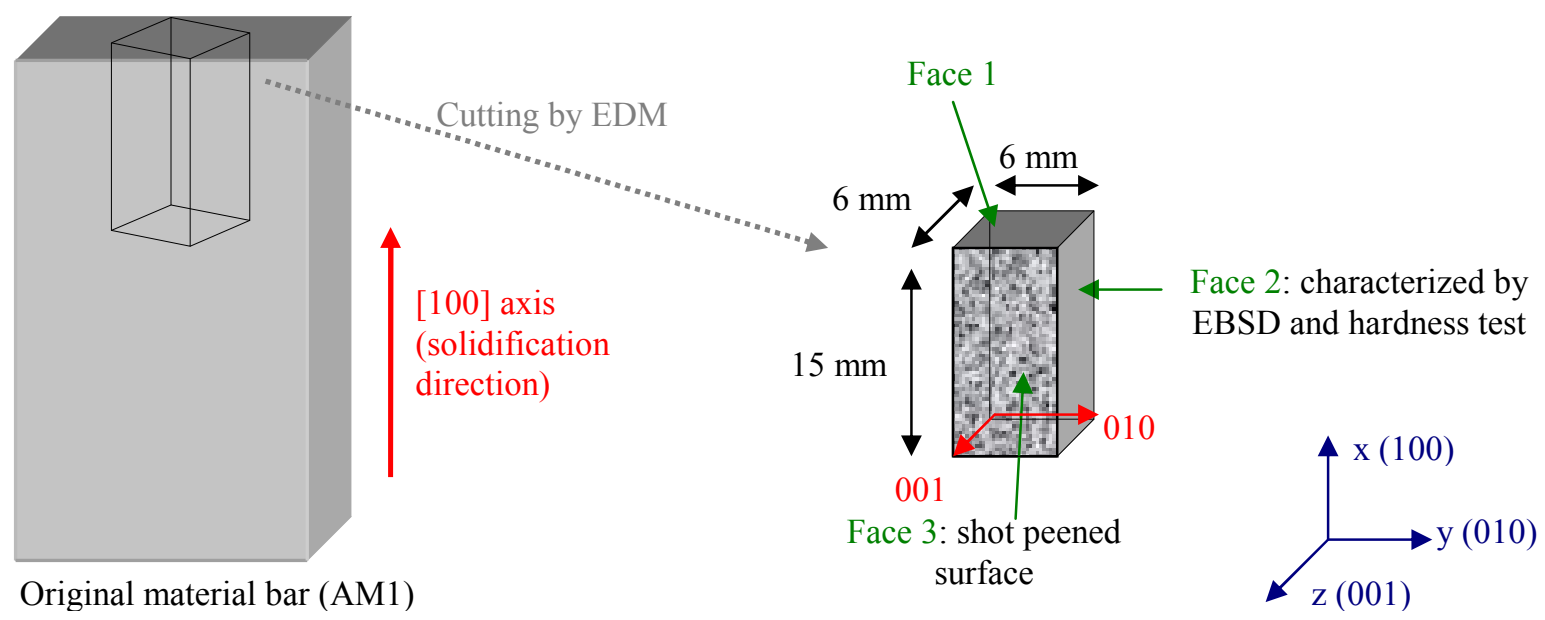

Fig 1. (a) Original material bar produced by directional solidification. (b) Sample dimensions and crystallographic directions.

Samples with faces oriented along the [100] crystallographic directions are removed using Electro Discharge Machining (EDM) from an AM1 bar produced by directional solidification at SNECMA (Fig 1). After a mechanical polishing by hand to remove the surface layer affected by the EDM, samples go through solution and aging heat treatments. At the millimeter scale, the optical microscopy observations reveal a microstructure made of dendrites. The primary dendrite arms which are oriented along the solidification direction are separated by $450-550 \mu \mathrm{m}$ and the secondary dendrite arms spacing is close to $50 \mu \mathrm{m}$ (Fig 2a). The longitudinal axe of the samples and the primary dendrites growth axis correspond to a [100] crystallographic direction. Fig $2 \mathrm{~b}$ shows the dendrite arrangement in the plane normal to solidification direction, which corresponds to "face 1" in Fig 1. At the microscopic scale, the microstructure is made of an austenitic nickel rich $\gamma$ matrix strengthened by about $70 \%$ volume fraction of $\gamma^{\prime}-\mathrm{Ni}_{3} \mathrm{Al}$ ordered precipitates with a cubic $\mathrm{L}_{2}$ structure. The $\gamma^{\prime}$ precipitates have cubical shapes. They are homogeneously distributed in the $\gamma$ matrix and have sizes of less than $1 \mu \mathrm{m}$. The $\gamma^{\prime}$ phase is highly coherent with the $\gamma$ matrix, since the mismatch between the lattice parameters of the two phases is smaller than $2 \%$.

A rectangular face of several samples, denoted by "face 3" in Fig 1, is shot-peened by an ultrasonic technique with 100C6 steel shots of $1 \mathrm{~mm}$ in diameter, at $100 \%$ coverage. Microhardness tests and scanning electron microscopy are used to characterize the zone affected by the shotpeening and determine the depth of the layers which will be removed by electro-polishing between two X-ray measurements.
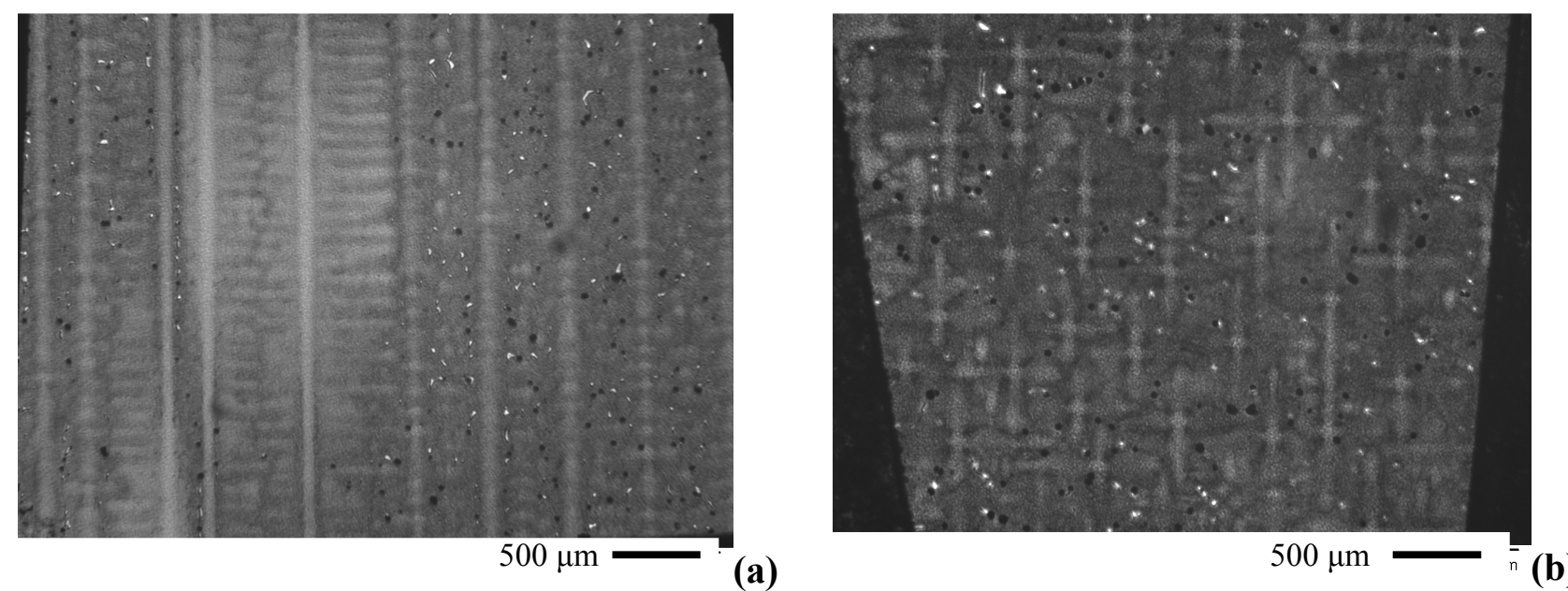

(a)

Fig 2. Sample surfaces optical images after chemical etching before shot-peening. (a) face 3 -(b) face 1 (see Fig 1.) 


\section{Microhardness and EBSD characterizations}

Hardness tests are realized with a Buelher OmniMet testing machine using a $0.025 \mathrm{~kg}$ load. Fig 3 shows the Vickers Hardness ( $\mathrm{VH}$ ) measured on a distance of $450 \mu \mathrm{m}$ along $\mathrm{z}$ axis on the "face 2" of a sample (Fig 1). The VH initially at 570, slightly increases from the sample surface to reach its maximum of 600 at $35 \mu \mathrm{m}$ (distance from the edge). Then, it decreases to 355 and reaches a plateau after $115 \mu \mathrm{m}$.

Analyses using Electron Back Scattering Diffraction (EBSD) are realized with a DSM960 system on a $440 \times 250 \mu \mathrm{m}^{2}$ zone using $1 \mu \mathrm{m}$ steps. Fig 4 represents the orientation map recorded on the "face $2 "$ of a sample after shot-peening. The crystalline disorientation, which was initially lower than $1^{\circ}$ in the core of the sample, is, in average, close to $4^{\circ}$ in the $60 \mu \mathrm{m}$ surface layer. The disorientation field at a given depth is heterogeneous since locally, disorientations range from $0^{\circ}$ to $8^{\circ}$. Microhardness and EBSD characterizations show that the depth affected by shot-peening is close to $100 \mu \mathrm{m}$.

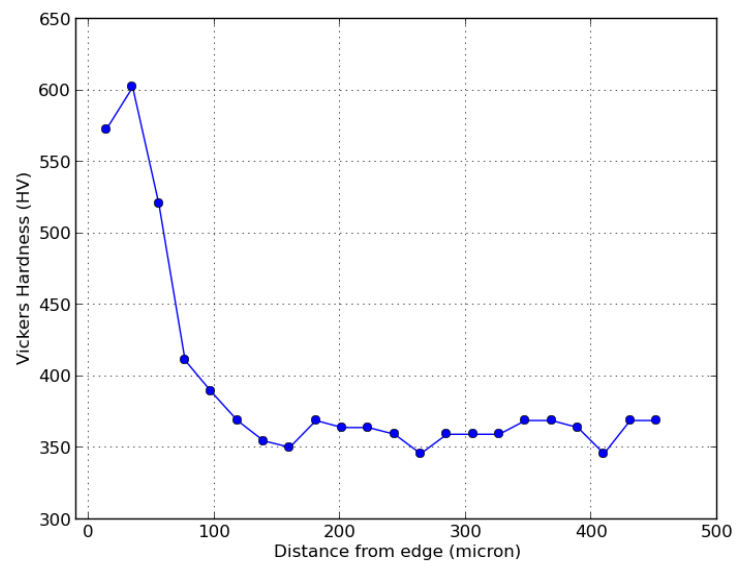

Fig 3. Vickers hardness as a function of depth.

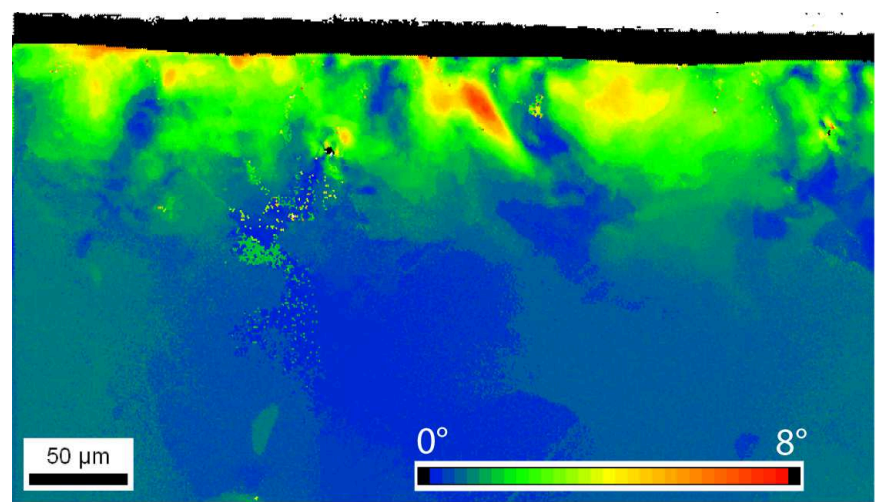

Fig 4. EBSD map of crystal orientations recorded on the "face $2 "$ of a sample (see Fig 1) after shot-peening. The surface of the sample is located at the top of the image.

\section{X-ray diffraction measurements}

The diffraction measurements are realized using a diffractometer composed of a microfocus generator equipped with a copper rotating anode, parabolic multilayer optics, a general purpose 6circles goniometer and a scintillation detector. The section of the incident beam is $1.4 \times 1.4 \mathrm{~mm}^{2}$ at the sample position and its divergence is about $1 \mathrm{mrad}$ in the measuring plane. The scintillation counter is located at $700 \mathrm{~mm}$ from the sample position. Diffusion and detector slits opening is set to $1 \times 1 \mathrm{~mm}^{2}$ in order to have a good signal to noise ratio for the high angle diffraction peaks recorded with the shot-peened sample. The structure factors associated with the $\gamma$ and $\gamma$ ' phases of the AM1 superalloy are listed in Table 2 for the diffraction peaks with $90^{\circ}<2 \theta<180^{\circ}$ for $\mathrm{CuK} \alpha$. Due to the high degree of coherence between the $\gamma$ and $\gamma^{\prime}$ phases, it is difficult to separate the two contributions from the signal measured with the fundamental diffraction peaks (Miller indices in black), especially when the peak width is broadened by crystal defects. The superstructure peaks (Miller indices in blue) are sensitive only to the $\gamma^{\prime}$ phase. In principle, strains tensors could be evaluated from these peaks. However, in the present case, this is not possible for shot-peened samples with our diffractometer due to the low values of the $\gamma^{\prime}$ structure factors, to the presence of dendrites, which distribute the intensity on several diffraction peaks ([5] and Fig 5b). In addition, the decrease of peak height when considering shot-peening samples is also a limiting factor (Fig 5a). A better analysis of $\gamma^{\prime}$ strains requires a higher X-ray flux. As a consequence, for shot-peened samples, strains tensors are evaluated from the fundamental peaks and the resulting residual stresses correspond to macrostrains averaged on the $\gamma$ and $\gamma^{\prime}$ phases. 
Table 1. AM1 structure factors (in electron unit) for the $\gamma$ and $\gamma^{6}$ phases, calculated from the results of 3D atom probe measurements.[6]. Only Miller indices corresponding to diffraction peaks with $90^{\circ}<2 \theta<180^{\circ}$ are given. The values in black correspond to the peaks associated with the face centered cubic lattice (fundamentals peaks).

The values in blue correspond to the additional peaks associated with the $\gamma^{6}$ phase (superstructure peaks).

\begin{tabular}{|c|c|c|c|c|c|c|c|c|c|}
\hline hkl & $\mathbf{3 1 1}$ & $\mathbf{2 2 2}$ & $\mathbf{3 2 0}$ & $\mathbf{3 2 1}$ & $\mathbf{4 0 0}$ & $\begin{array}{c}\mathbf{4 1 0} \\
\mathbf{3 2 2}\end{array}$ & $\begin{array}{c}\mathbf{4 1 1} \\
\mathbf{3 3 0}\end{array}$ & $\mathbf{3 3 1}$ & $\mathbf{4 2 0}$ \\
\hline $\mathbf{2} \boldsymbol{\theta}$ & $90.63^{\circ}$ & $95.90^{\circ}$ & $101.23^{\circ}$ & $106.66^{\circ}$ & $118.07^{\circ}$ & $124.23^{\circ}$ & $130.87^{\circ}$ & $138.27^{\circ}$ & $146.95^{\circ}$ \\
\hline $\boldsymbol{\gamma}$ & 57.7 & 55.8 & & & 48.9 & & & 45.3 & 44.6 \\
\hline $\boldsymbol{\gamma}^{\prime}$ & 55.9 & 54 & 1.8 & 1.7 & 47.4 & 1.5 & 1.4 & 43.9 & 43.3 \\
\hline
\end{tabular}

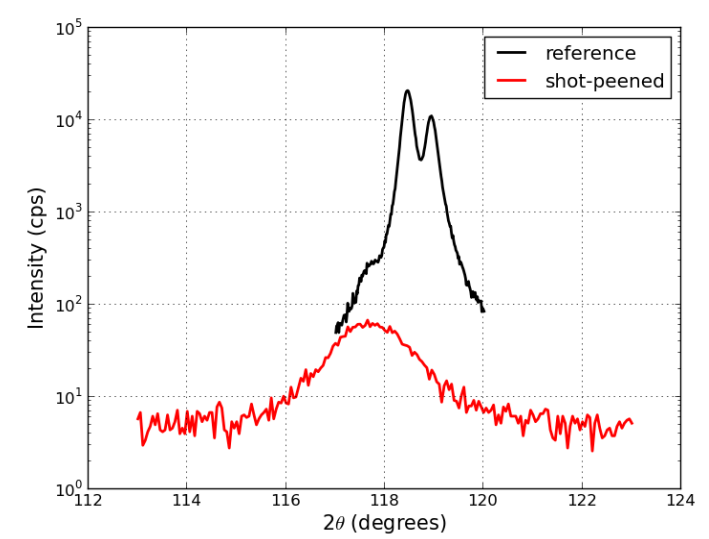

(a)

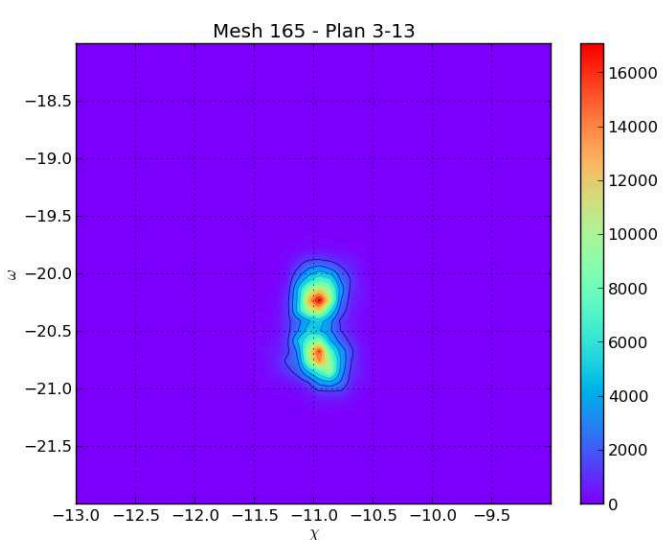

(b)

Fig 5. (a) (004) diffraction peak measured at the surface of a sample before and after shot peening. (b) Intensity map recorded for the (3-13) lattice planes by rocking the sample with the $\chi$ and $\omega$ motors, while the detector position is kept fixed.

In order to determine the residual stresses in the sample frame, the cell parameters in the reference state, the elasticity tensor of the material and the orientation matrix must be known. The cell parameters consist in 3 lattice parameters and 3 angles between the axes. They enable to determine the 6 components of the metric tensor $\mathrm{G}^{*}$ of the space defined by the reciprocal lattice. The $\gamma$ and $\gamma^{\prime}$ phases have both the cubic symmetry. Consequently, the reference state is chosen as a cubic cell with a lattice parameter $\mathrm{a}_{0}$. The corresponding metric tensor $\mathrm{G}_{0} *$ is purely hydrostatic. The AM1 elasticity tensor have been measured by SNECMA at $20^{\circ} \mathrm{C}$ using tensile tests: $\mathrm{C}_{1111}=296 \mathrm{GPa} ; \mathrm{C}_{1122}=204 \mathrm{GPa} ; \mathrm{C}_{1212}=125 \mathrm{GPa}$ [7]. At the present time, the elastic modulus of the $\gamma$ and $\gamma^{\prime}$ phases are unknown. The orientation matrix converts the components of the residual stress tensor calculated with Hooke's law, from the crystal frame ([100], [010], [001]) to the sample frame $(\mathrm{x}, \mathrm{y}, \mathrm{z})$. In this study, the two reference frames are almost confounded, as shown in Fig. 1. The components of the residual stress tensor in the sample frame are thus almost equal to those in the crystal frame. The lattice plane spacing $\mathrm{d}_{\mathrm{hkl}}$ are measured using Bragg's law. They are related to the 6 components of the metric tensor $\mathrm{G}^{*}$ of the strained reciprocal space by the equation [3]:

$$
d_{h k l}^{-2}=h^{2} g_{11}^{*}+k^{2} g_{22}^{*}+l^{2} g_{33}^{*}+2 h k g_{12}^{*}+2 h l g_{31}^{*}+2 k l g_{23}^{*}
$$

which can be written in the matrix form: $\left[\mathrm{G}^{*}\right] \cdot[\mathrm{H}]=\left[\mathrm{D}^{-2}\right]$. Thus, at least 6 distances of non collinear lattice planes must be measured to fully determine tensor $\mathrm{G}^{*}$. In practice, about 20 diffraction peaks are recorded and the vector which contains the 6 independent components of the metric tensor is given by a least square regression with the Moore-Penrose pseudo-inverse:

$$
\left[\mathrm{G}^{*}\right]=\left(\left[\mathrm{H}^{\mathrm{T}}\right] \cdot[\mathrm{H}]\right)^{-1} \cdot\left[\mathrm{H}^{\mathrm{T}}\right] \cdot\left[\mathrm{D}^{-2}\right]
$$


Finally, the strain tensor $[\varepsilon]$ is calculated from Ortner's equation : $[\varepsilon]=\left(\mathrm{a}_{0} / 2\right) .\left(\left[\mathrm{G}_{0} *\right]-\left[\mathrm{G}^{*}\right]\right)$ where $\mathrm{a}_{0}$ is the lattice parameter of the stress-free reference state. The angular position of 21 diffraction peaks corresponding to the highest diffraction angles are used to calculated the strain tensor (see Fig 6). The position of each peak is defined using a least square fitting procedure where the peaks are modeled with a Pseudo-Voigt function taking into account the $\mathrm{K} \alpha_{1}-\mathrm{K} \alpha_{2}$ doublet through Rachinger's assumptions [8].

The lattice parameter of the reference state is chosen from measurements on a sample which has not been shot-peened. We found the value $\mathrm{a}_{0}=0.35880 \mathrm{~nm}$ which corresponds to $\sigma_{\mathrm{zz}}=0$. This parameter is chosen constant for all layers studied. Another possible hypothesis would have been to take $\sigma_{\mathrm{zz}}=0$ for all layers (commonly used) but this assumption is debatable when we have a biphase material as it is the case here.

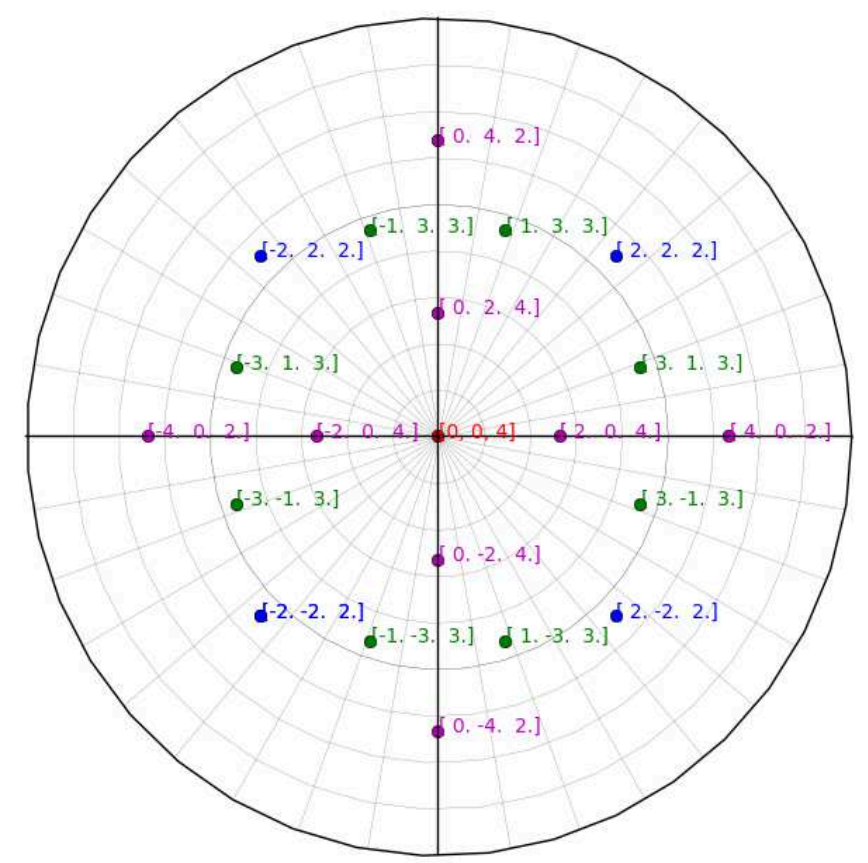

Fig 6. Stereographic representation, in the crystal frame, of the lattice planes used for residual stress determination (for fundamental reflections).

Fig 7 shows the evolution of each component of the stress tensor (in the sample frame) versus depth for a shot-peened sample. The major contributions arise from $\sigma_{\mathrm{xx}}$ and $\sigma_{\mathrm{yy}}$, which are almost equal. The values of $\sigma_{\mathrm{yz}}, \sigma_{\mathrm{xz}}$ and $\sigma_{\mathrm{xy}}$ are smaller than $35 \mathrm{MPa}$. This profile is representative of shotpeened specimens with a maximum of $1100 \mathrm{MPa}$ compressive stresses reached at $17 \mu \mathrm{m}$ below the surface. Stress uncertainties are estimated from the errors of peak fitting which are propagated using Monte Carlo loops.

Thus, the thickness affected by the shot-peening treatment can be evaluated in a consistent way through four different indicators: about $130 \mu \mathrm{m}$ according to the residual stress profile (Fig 7); about $120 \mu \mathrm{m}$ according to peak width, indicating crystalline defects (Fig 8); about $60 \mu \mathrm{m}$ from EBSD observation giving the mosaic structure (disorientations) of the crystal (Fig 4) and about $100 \mu \mathrm{m}$ from microhardness tests which can be influenced by the 3 previous phenomena (Fig 3) 


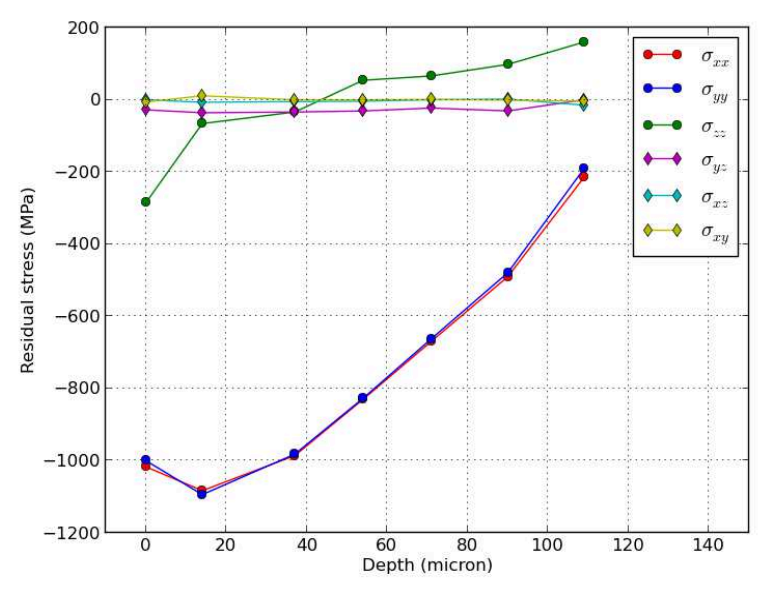

Fig 7. Residual stress as a function of depth in a shotpeened sample. Uncertainties are estimated between 6 and $10 \mathrm{MPa}$

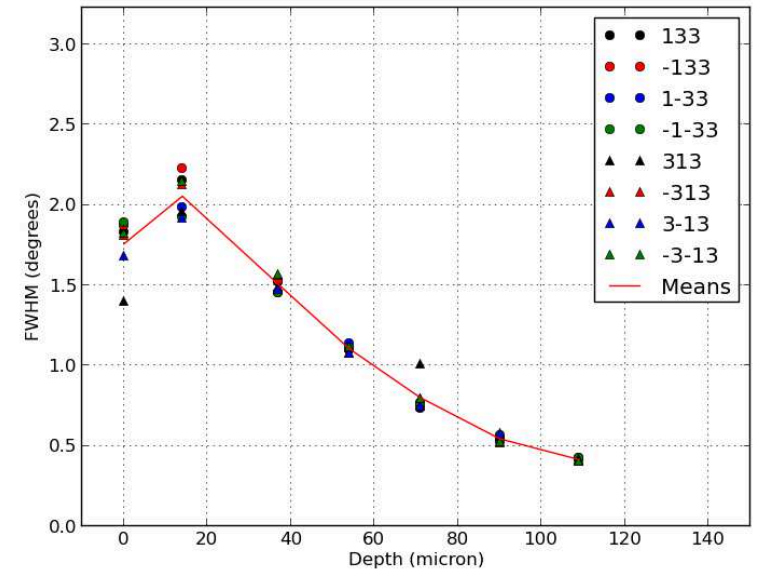

Fig 8. FWHM vs. depth for (133) lattice planes.

\section{Conclusions}

A method proposed by Ortner was used to determine residual stresses in an AM1 nickel superalloy single crystals. A profile over $110 \mu \mathrm{m}$ has been determined on a specimen shot-peened by an ultrasonic technique, on a surface parallel to (100) plane. It was shown that the shot-peening treatment induces residual stresses in a surface layer of about $130 \mu \mathrm{m}$ thickness, with a maximum of $1100 \mathrm{MPa}$ reached at $17 \mu \mathrm{m}$ beneath the surface. In addition, the hardened depth due to shot peened is estimated between $115 \mu \mathrm{m}$ and $125 \mu \mathrm{m}$.

\section{References}

[1] B.J. Foss, S. Gray, M.C. Hardy, S. Stekovic, D.S. McPhail and B.A. Shollock, "Analysis of shot-peening and residual stress relaxation in the nickel-based superalloy RR1000", Acta Materialia, 61 (2013) p. 2548-2559.

[2] Y. Chen and C. Jiang, "Effect of shot peening on surface characteristics of Ni-Based SingleCrystal Superalloy”, Materials Transactions, 54 (2013) p. 1894-1897.

[3] B. Ortner, «Röntgenographische Spannungsmessung an Einkristallinen proben », Eigenspannungen, Karlsruhe, (1983), Bd2, p46-68

[4] M. François. Analyse des contraintes résiduelles dans le monocristaux et les matériaux à gros grains par diffraction des rayons X, mémoire de DEA, ENSCP-Univ. Paris VI, (1987).

[5] U. Brückner, A. Epishin, T. Link and K. Dressel. "The influence of dendritic structure on the $\gamma / \gamma^{\prime}$-lattice misfit in the single-crystal nickel-base superalloy CMSX-4", Materials Science and Engineering A247 (1998) 23-31.

[6] A. Buchon, S. Chambreland and D. Blavette. "Proceedings of Colloque National Superalliage Monocristallin”, Nancy, France, (1990), p. 129 -140

[7] L. Espié, «Etude expérimentale et modélisation numérique du comportement de monocristaux de superalliages ». PhD Thesis, Ecole Nationale Supérieure des Mines de Paris, (1996).

[8] W.A. Rachinger, "A correction for the alpha-1 alpha-2 doublet in the measurement of widths of x-ray diffraction lines”, Journal of Scientific Instruments, 25 (1948) p 254-259. 